\title{
Fetal and Neonatal Alloimmune Thrombocytopenia: Harvesting the Evidence to Develop a Clinical Approach to Management
}

\author{
Amanda Symington, M.H.Sc., ${ }^{1}$ and Bosco Paes, M.B.B.S., F.R.C.P.I., F.R.C.P.C. ${ }^{1,2}$
}

Neonatal alloimmune thrombocytopenia (NAIT) is the most common cause of severe thrombocytopenia in an otherwise healthy newborn. The most serious complication is intracranial hemorrhage, which can occur either in the fetus or the newborn. Despite the known serious sequelae, both antenatal management and neonatal treatment modalities are plagued by the lack of gold standard evidence to appropriately direct therapy. Maternal, risk-based therapeutic approaches range from invasive protocols to relatively benign noninvasive strategies to avoid serious procedural complications. Intravenous immunoglobulin (IVIG) with or without steroids and fetal blood sampling constitute the mainstay of antenatal management. Neonatal interventions principally focus on the use of antigennegative compatible or random donor platelets and IVIG. While awaiting the results of controlled trials, each institution must develop a standardized, collaborative, multidisciplinary approach to the screening, diagnostic evaluation, and management of unexpected and anticipated NAIT based on experience, product availability, and emerging scientific data.

KEYWORDS: Maternal, fetal, neonatal, alloimmune thrombocytopenia, management

Neonatal alloimmune thrombocytopenia (NAIT) is the most common cause of moderately severe thrombocytopenia in an otherwise healthy newborn infant. ${ }^{1}$ NAIT results from a platelet antigen incompatibility between the mother and the fetus and is analogous to Rhesus hemolytic disease. Typically, unanticipated NAIT is first diagnosed at or shortly after delivery, with the infant presenting with a platelet count $<50 \times 10^{9} / \mathrm{L}$. For some infants, the thrombocytopenia leads to multiple platelet transfusions, intracranial hemorrhage (ICH) and significant long-term neurological deficits. This article will focus on human platelet alloantigen (HPA)-1a alloimmunization, the pathogenesis and incidence of NAIT, as well as the antenatal and postnatal management of this condition.

\section{MATERIALS AND METHODS}

A systematic literature search was utilized to retrieve the studies and articles for this review. An electronic MEDLINE literature search was performed using the following terms: neonatal OR fetomaternal OR newborn
${ }^{1}$ Hamilton Health Sciences, McMaster Children's Hospital (Neonatal Division), and ${ }^{2}$ Department of Pediatrics (Division of Neonatology), McMaster University, Hamilton, Ontario, Canada.

Address for correspondence and reprint requests: Bosco A. Paes, M.B.B.S., F.R.C.P.I., F.R.C.P.C., McMaster Children's Hospital (Room 3A), 1200 Main Street West, Hamilton, Ontario L8S 4J9, Canada (e-mail: paes@mcmaster.ca).
Am J Perinatol 2011;28:137-144. Copyright (C) 2011 by Thieme Medical Publishers, Inc., 333 Seventh Avenue, New York, NY 10001, USA. Tel: +1(212) 584-4662.

Received: April 17, 2010. Accepted after revision: June 30, 2010. Published online: August 10, 2010.

DOI: http://dx.doi.org/10.1055/s-0030-1263296.

ISSN 0735-1631. 
AND alloimmune thrombocytopenia OR NAIT. The Cochrane Central Register of Controlled Trials (Cochrane Library issue 1, 2010) was searched for controlled studies and the Cochrane Database of Systematic Reviews (Cochrane Library issue 1, 2010) was searched for systematic reviews on the antenatal and postnatal management of NAIT. No language restriction was applied. All identified reports were checked for references to additional studies.

\section{Pathogenesis of NAIT}

HPAs are proteins found on the surface of platelets, and the HPA system is used in the nomenclature of platelet antigens. An alloantigen is an antigen that is present in a portion of the population and absent in the rest of that population. There are 24 platelet-specific alloantigens that have been recognized, and they are numbered according to when they were first described. ${ }^{2}$ Of the platelet alloantigens, 12 are grouped into biallelic systems (HPA-1, -2, -3, -4, -5, -15). The platelet alloantigen is then further defined according to the frequency in which it appears in the population. A high-frequency alloantigen is designated "a" and a low-frequency alloantigen is designated "b." "HPA-1a is the most common platelet alloantigen in the Caucasian population and is responsible for $\sim 80 \%$ of the cases of NAIT.

NAIT can result when there is an incompatibility between the platelet antigen of the mother and her fetus. Maternal anti-platelet immunoglobulin G (IgG) alloantibodies, produced after exposure to the foreign platelet antigen, cross the placenta and bind to the fetal platelets. Therefore, a mother with HPA-1a-negative platelets who has a fetus with HPA-1-positive platelets becomes sensitized to the HPA-1a-positive platelets and produces anti-HPA-1a, anti-platelet IgG. The antibodycoated platelets pass through the reticuloendothelial system and are rapidly removed from the circulation, resulting in fetal thrombocytopenia. Expression of HPA-1a antigens on fetal platelets has been demonstrated as early as 16 weeks' gestation; however, the first appearance of HPA-1a antibodies may only become evident in the fetus after 20 weeks. ${ }^{3}$ The presence of antibodies to the HPA-1a antigen is also strongly associated with the HLA DRB3*0101. ${ }^{4-9}$

Several factors may influence the severity of the thrombocytopenia; these include: the concentration and subclass of maternal $\mathrm{IgG}$ alloantibodies, the density of the "target" antigens on the fetal platelets, the activity of phagocytes in the fetal reticuloendothelial system, and the ability of the fetal bone marrow to compensate for the accelerated destruction of antibodysensitized platelets. ${ }^{10}$

Ninety-eight percent of Caucasian females have the genotype HPA- $1 \mathrm{a} / 1 \mathrm{a}^{7}$ and approximately $2 \%$ are HPA-1a-negative or homozygous HPA-1b/1b. If the mother of an infant with NAIT is HPA- $1 \mathrm{~b} / 1 \mathrm{~b}$ the father will either be heterozygous, HPA-1a/1b, or homozygous HPA-1a/1a. If the father has the genotype HPA- $1 \mathrm{a} / 1 \mathrm{~b}$ then $50 \%$ of his offspring will be at risk for NAIT and $100 \%$ of his offspring will be at risk for NAIT if his genotype is HPA-1a/1a.

Although the pathogenesis of NAIT is similar to Rhesus hemolytic disease of the newborn, in NAIT, the first pregnancy can be affected, and there is a high recurrence risk of fetal/neonatal thrombocytopenia (FNAIT) in future pregnancies.

\section{Incidence}

The frequency of HPAs varies across the world. The HPA-1a that predominates in the Caucasian population is rare in the Asian population, where HPA-5b incompatibility is the most common cause of fetomaternal alloimmune thrombocytopenia. ${ }^{11}$

The incidence of HPA-1a-negative mothers can be derived from the large prospective trials. A summary of these trials is shown in Table 1. Ten in 1000 mothers were found to be HPA-1a-negative, $10 \%$ were alloimmunized to the HPA-1a antigen, and $40 \%$ of these mothers delivered infants with NAIT. ${ }^{4-9,12-14}$ The overall incidence of NAIT is 0.7 in 1000 pregnancies.

Thrombocytopenia is defined as a platelet count $<150 \times 10^{9} / \mathrm{L}$. The incidence of thrombocytopenia in large prospective studies of unselected newborns is 0.5 to $0.9 \%{ }^{15-17}$ and $0.12 \%$ will be severely thrombocytopenic with a platelet count of $<50 \times 10^{9} / \mathrm{L}^{1,16}$ In NAIT, 50 to $80 \%$ will have platelet counts $<50 \times 10^{9} / \mathrm{L}^{8,16,18}$ Sensitization to HPA-1a is the most common cause of severe alloimmune thrombocytopenia and is responsible for 75 to $85 \%$ of the cases. ${ }^{10,18,19}$ Bussel et a $1^{19}$ reported an initial median platelet count of $18 \times 10^{9} / \mathrm{L}$ in infants born with an HPA-1a incompatibility as compared with $60 \times 10^{9} / \mathrm{L}$ with other antigen incompatibilities.

The incidence of an $\mathrm{ICH}$ as a result of alloimmunization to the HPA-1a antigen is $\sim 20 \%{ }^{20}$ The majority of ICHs occur antenatally (80\%), with $14 \%$ of these hemorrhages occurring before 20 weeks' gestation and $28 \%$ before 30 weeks' gestation. ${ }^{20}$

\section{Clinical Presentation and Predictors of Thrombocytopenia}

The diagnosis of NAIT in the firstborn child is usually unexpected by the family and health care providers and is frequently made at birth. The infant can present with petechiae, bruising, and bleeding but will otherwise look well. The treatment of this infant is discussed later in this article. The subsequent pregnancies of this mother can then be closely followed. 
Table 1 Prospective Studies of Alloimmunization to HPA-1a Antigen in Nonselected Pregnancies

\begin{tabular}{|c|c|c|c|c|c|c|}
\hline Authors & Year & $\begin{array}{l}\text { Total No. } \\
\text { of Women } \\
\text { Typed }\end{array}$ & $\begin{array}{l}\text { No. of HPA-1a- } \\
\text { Negative } \\
\text { Women }\end{array}$ & $\begin{array}{l}\text { No. of Women with } \\
\text { anti-HPA-1a/No. } \\
\text { Assessed }\end{array}$ & $\begin{array}{l}\text { No. of Cases of } \\
\text { NAIT (Platelet } \\
\text { count }<150 \times 10^{9} / \mathrm{L} \text { ) }\end{array}$ & $\begin{array}{l}\text { No. of Cases } \\
\text { with Intracranial } \\
\text { Hemorrhage }\end{array}$ \\
\hline Mueller-Eckhardt et al ${ }^{12}$ & 1985 & 1211 & 26 & $2 / 26$ & 2 & 0 \\
\hline Blanchette et al $\left.\right|^{4}$ & 1990 & 5000 & 81 & $3 / 50$ & 1 & 1 \\
\hline Doughty et $\mathrm{al}^{5}$ & 1995 & 3473 & 74 & $2 / 71$ & $2^{*, \dagger}$ & 0 \\
\hline Durand-Zaleski et al ${ }^{6}$ & 1996 & 2066 & 52 & $4 / 45^{\ddagger}$ & 1 & 0 \\
\hline Williamson et $\mathrm{al}^{9}$ & 1998 & 24,417 & 618 & $46 / 387$ & $19^{\S}$ & 1 \\
\hline Davoren et al ${ }^{13}$ & 2003 & 4090 & 54 & $3 / 34$ & 3 & 0 \\
\hline Maslanka et al ${ }^{14}$ & 2003 & 8013 & 144 & $12 / 122$ & $3^{\| 1}$ & 1 \\
\hline Turner et $a l^{8}$ & 2005 & 25,506 & 546 & $25 / 318$ & 8 & 2 \\
\hline Kjeldsen-Kragh et al ${ }^{7}$ & 2007 & 100,448 & 2111 & $210 / 1990$ & 85 & 2 \\
\hline
\end{tabular}

*Twins.

† One mother treated with IVIG. Platelet count prior to delivery $210 \times 10^{9} / \mathrm{L}$.

$\ddagger$ One mother treated with IVIG. Platelet count at birth $207 \times 10^{9} / \mathrm{L}$. One mother treated with corticosteroids (two mothers did not consent to fetal blood sampling).

Excludes one in utero death.

"One case treated with intrauterine platelet transfusions, one case treated with intrauterine platelet transfusions and IVIG. Platelet count at birth $237 \times 10^{9} / \mathrm{L}$. ICH occurred despite intrauterine transfusions.

HPA, human platelet alloantigen; ICH, intracranial hemorrhage; IVIG, intravenous immunoglobulin; NAIT, neonatal alloimmune thrombocytopenia.

In a recent prospective study, Killie et $\mathrm{al}^{21} \mathrm{dem}-$ onstrated a strong correlation between maternal antibody levels and the platelet count of the neonate. Maternal anti-HPA-1a antibody levels above $3.0 \mathrm{IU} /$ $\mathrm{mL}$ at 22 and 34 weeks' gestation were good predictors of the degree of thrombocytopenia in the neonate both in the first and subsequent pregnancies with a clinical sensitivity of $93 \%$ and specificity of $63 \%{ }^{22}$

The best noninvasive predictor of the severity of thrombocytopenia and related ICH in future pregnancies with HPA-1a alloimmunization is the presence of an in utero $\mathrm{ICH}$ in the sibling. ${ }^{19}$ The rate of recurrence of an ICH in a sibling of an infant with NAIT and ICH is $72 \%$ with fetal deaths not included in the analysis and $79 \%$ when fetal deaths were included. ${ }^{23}$ Unfortunately, there are no other noninvasive surrogate measures to determine the severity of thrombocytopenia in fetuses whose sibling did not have an ICH.

The risk of having an alloimmunized fetus can be guided by the presence of HLA DRB3*0101, which is linked to HPA-1a and has a positive predictive value of $35 \%$ and a negative predictive value of $99.6 \% .{ }^{9}$ However, this investigation is limited to women who have had a previously affected fetus.

\section{Antenatal Management}

Routine screening to identify fetuses at risk for hemolytic disease of the newborn is well established. A similar screening program of all pregnant women to identify those fetuses at risk for NAIT is not routinely performed. The lack of a standardized approach to the antenatal management of these pregnancies has impacted the adoption of a universal screening program. ${ }^{24}$ A typical screening and intervention program encompasses HPA- 1a typing of all pregnant women antenatally and screening HPA-1a-negative individuals for anti-HPA-1a antibodies at $\sim 22$ and 34 weeks' gestation. Immunized women are offered delivery by cesarean section 2 to 4 weeks prior to term, and infants with severe thrombocytopenia (platelet count $\left.<35,000 \times 10^{9} / \mathrm{L}\right)$ are immediately treated with reserved, donor platelets, which are compatible in $>95 \%$ of all cases, to reduce the severity of complications $^{25}$ (Fig. 1). Discussion is ongoing on the costeffectiveness of implementing such programs and whether the short- and long-term outcomes will be favorable as reported by Kjeldsen-Kragh in Norway. ${ }^{6-8,26,27}$ The cost of a screening program for primigravida women to prevent one case of death/disability was estimated at $\$ 1,876,656$ in $2005 .^{8}$ More recently, a cost-utility analyses by Killie et $\mathrm{al}^{27}$ and Kjeldsen-Kragh et $\mathrm{al}^{25}$ showed that antenatal screening would reduce health care costs by almost $€ 1.7$ million, would generate between 210 and 230 additional quality-adjusted lifeyears, and is being considered for adoption as part of the antenatal program in Norway.

A mother with an identified personal or family history of NAIT allows health care professionals to provide close surveillance and early antenatal management during the pregnancy. A maternal sister who has had a pregnancy that has been complicated by NAIT should alert the physician to proceed with platelet antigen typing in her sibling's pregnancy. New cases of NAIT, with a negative family history, are unlikely to be recognized prior to delivery. If an in utero ICH or its sequelae such as cysts, leukomalacia, or hydrocephalus is identified, then NAIT should be strongly considered as the precipitating cause. If FNAIT is suspected, then identification of the maternal HPA antibody and serological platelet phenotyping/genotyping of the parents for a 


\section{Algorithm for the Management of Unexpected Alloimmune Neonatal Thrombocytopenia (NAIT)}

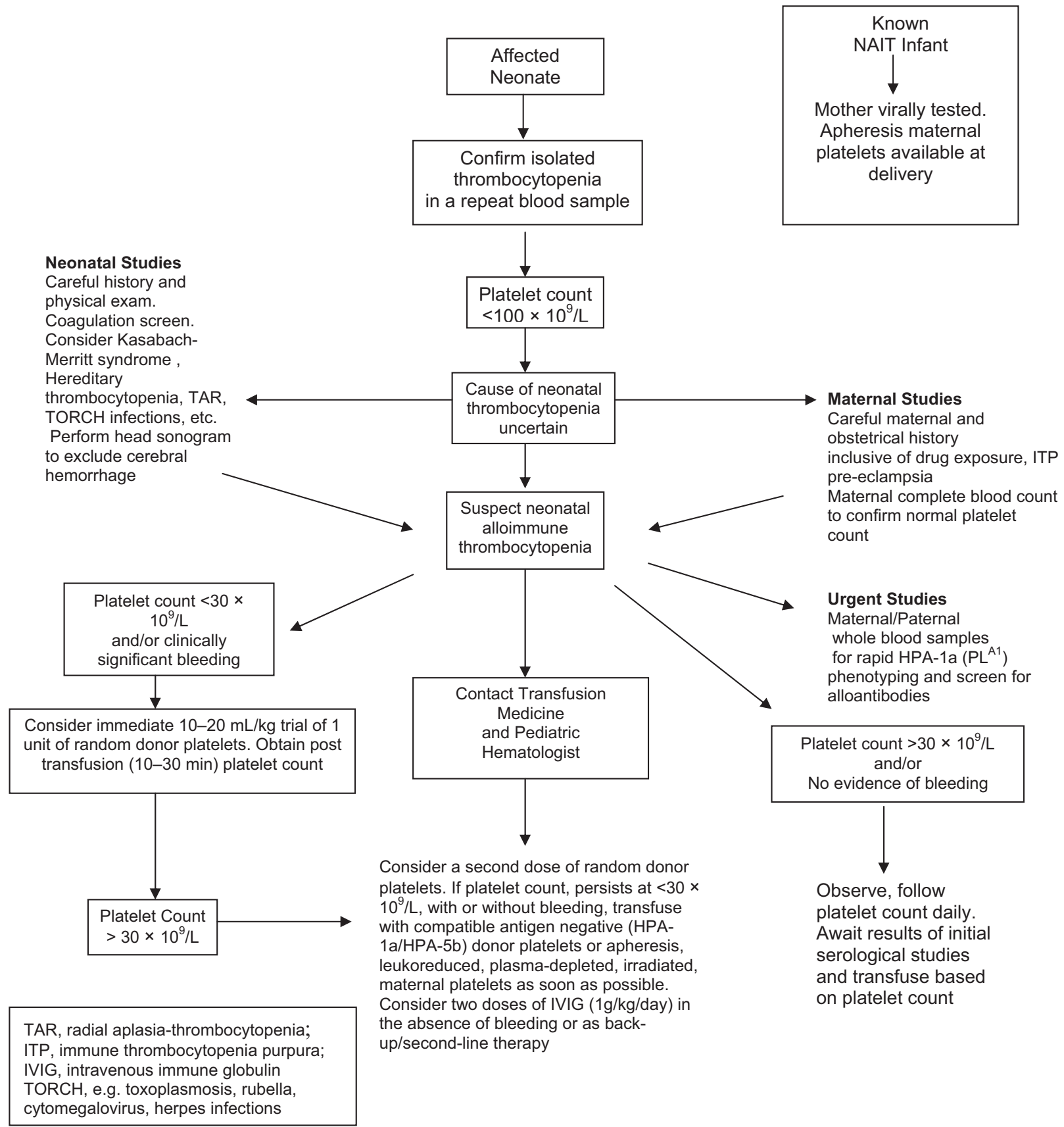

Figure 1 Algorithm for the management of unexpected alloimmune neonatal thrombocytopenia (NAIT). (Adapted from Blanchette VS, Johnson J, Rand M. The management of alloimmune neonatal thrombocytopenia. Baillière's Clin Haematol 2000;13:365-390, with permission from Elsevier.)

potential platelet incompatibility is essential to confirm the diagnosis. Commercial enzyme-linked immunosorbent antibody kits (Pak-Plus or Pak12, GTI Diagnostics, Waukesha, WI) are now available for initial screens followed by the monoclonal antibody-specific immobilization of platelet antigens assay and radioimmunoprecipitation assay (RIP) for further antibody testing. ${ }^{28,29}$ The RIP assay is able to identify all platelet glycoproteins including previously uncharacterized antigen targets, but its use is limited by cost, the need for specialized expertise, and the use of radioisotopes. ${ }^{29}$ Amniocentesis can be employed for fetal-maternal antigen testing if the father is heterozygous or in the case of doubtful paternity.

The goal of antenatal therapy is to reduce the severity of thrombocytopenia and thereby reduce the complications of ICH, long-term disability, and death. 
Options for therapy include in utero transfusions of antigen compatible platelets, intravenous immunoglobulin (IVIG), and systemic corticosteroids. Studies on which to base therapy are mainly observational because of the low incidence of alloimmunization to the HPA-1a antigen and NAIT in the general population.

In utero platelet-compatible transfusions have been shown to effectively increase the fetal platelet count, ${ }^{30-33}$ but weekly transfusions are necessary because of the short half-life of platelets, and there are significant risks to the procedure. In a retrospective review of 12 pregnancies and 84 platelet transfusions, Overton et $\mathrm{al}^{31}$ reported the procedure-related fetal loss to be $1.2 \%$ $(1 / 84)$ per procedure and $8.3 \%(1 / 12)$ per pregnancy. This was consistent with the experience of other centers. There were two deaths reported in the study; one was directly related to the procedure after the needle dislodged from the placental cord insertion site, and the other occurred 1 week following a successful in utero platelet transfusion. Neither of the two deaths was related to $\mathrm{ICH}$.

Quantification of the fetal platelet count, to evaluate effectiveness of treatment, can be done by cordocentesis, but this carries a risk of complications that include bleeding, infection, thrombosis, preterm delivery, and death. ${ }^{30,31,34-36}$ A 2003 review by Radder et $\mathrm{al}^{23}$ estimated the total complication rate for cordocentesis for NAIT to be $2.8 \%$.
IVIG and IVIG in combination with a corticosteroid have been used for several years as a preventive strategy in the management of FNAIT. Immunoglobulins play a major role in host defense include antigen binding and a variety of effector functions such as complement activation, complement binding, and binding to $\mathrm{Fc}$ receptors. ${ }^{37}$ However, their action in FNAIT has not been clearly delineated. Importantly, there is considerable intra- and interpopulation variation in the pharmacokinetics of IVIG. ${ }^{38}$ There have been no randomized control trials (RCTs) evaluating the efficacy of IVIG or steroids alone versus no treatment in alloimmune thrombocytopenia. Trials of this nature would be unethical because of the known risk of $\mathrm{ICH}$ with this condition. However, many observational reports comparing two different treatment modalities have been executed. Rayment et $\mathrm{al}^{11}{ }^{11}$ in a Cochrane systematic review, summarized the results of 26 observational studies on the maternal administration of corticosteroids and IVIG in pregnancies with fetomaternal alloimmune thrombocytopenia. The results of these studies are unclear with regard to the prevention of an ICH, particularly in the most severely affected fetuses. Three RCTs have examined the role of IVIG with or without steroids. ${ }^{39-41}$ Table 2 shows the outcomes of these trials. Bussel et $\mathrm{al}^{39}$ did not demonstrate any difference in the fetal platelet count between IVIG and IVIG with steroids. Moreover, Berkowitz et $\mathrm{al}^{40}$

Table 2 Randomized Controlled Trials of Intravenous immunoglobulin (IVIG) with or without Steroids in Pregnancies with Alloimmune Thrombocytopenia

\begin{tabular}{|c|c|c|c|c|c|c|c|c|}
\hline Author & Year & $\begin{array}{l}\text { Time } \\
\text { Frame }\end{array}$ & $\begin{array}{l}\text { Sample } \\
\text { Size }\end{array}$ & Intervention & $\begin{array}{l}\text { Nonresponders } \\
(n)\end{array}$ & $\begin{array}{r}\text { Mean C } \\
\text { Platelet Cou } \\
\text { First and Second }\end{array}$ & $\begin{array}{l}\text { hange in } \\
\text { Int Between } \\
\text { Samples }\left(10^{9} / \mathrm{L}\right)\end{array}$ & $\begin{array}{l}\text { Intracranial } \\
\text { Hemorrhage }\end{array}$ \\
\hline \multirow{2}{*}{$\begin{array}{l}\text { Bussel } \\
\text { et }^{339}\end{array}$} & 1996 & 1990-1993 & 54 & IVIG & 12 & 37,400 & & 0 \\
\hline & & & & $\begin{array}{l}\text { IVIG }+ \\
\quad \text { dexamethasone }\end{array}$ & $\begin{array}{l}\text { (Groups } \\
\text { combined) }\end{array}$ & 33,900 & & 0 \\
\hline \multirow{6}{*}{$\begin{array}{c}\text { Berkowitz } \\
\text { et al }\end{array}$} & 2006 & 1994-2001 & 79 & High risk*: & & & & \\
\hline & & & & IVIG & 16 & 17,300 & & 1 \\
\hline & & & & $\begin{array}{c}\text { IVIG }+ \\
\text { prednisone } \\
\text { Standard risk }{ }^{\dagger}\end{array}$ & 7 & 67,100 & & 0 \\
\hline & & & & IVIG & 10 & 30,600 & & $2^{\ddagger}$ \\
\hline & & & & Prednisone & 12 & 25,700 & & \\
\hline & & & & & & $\begin{array}{l}\text { Platelet Count } \\
\text { at } 32 \text { wk } \\
\left(n<30 \times 10^{9} / \mathrm{L}\right)\end{array}$ & $\begin{array}{l}\text { Platelet Count } \\
\text { at Birth } \\
\left(n<50 \times 10^{9} / \mathrm{L}\right)\end{array}$ & \\
\hline \multirow{2}{*}{$\begin{array}{c}\text { Berkowitz } \\
\text { et }\left.^{4}\right|^{41}\end{array}$} & 2007 & 2001-2006 & 73 & IVIG & & 9 & 5 & $1^{\S}$ \\
\hline & & & & IVIG + prednisone & & 5 & 4 & $1^{\S}$ \\
\hline
\end{tabular}

*High-risk—sibling with an $\mathrm{ICH}$ during the peripartum period or initial fetal platelet count $<20 \times 10^{9} / \mathrm{L}$.

†Standard risk-no sibling with an $\mathrm{ICH}$, initial fetal platelet count $20-100 \times 10^{9} / \mathrm{L}$

拉tribution between groups unclear.

${ }^{\S}$ Cases not treatment failures.

$\mathrm{ICH}$, intracranial hemorrhage; IVIG, intravenous immunoglobulin. 
were also not able to demonstrate a difference in standard risk pregnancies but did demonstrate that IVIG and prednisone was more effective in raising the fetal platelet count in high-risk pregnancies. The Berkowitz et $\mathrm{al}^{41}$ trial in 2007 showed good outcomes and comparable results between the IVIG and the IVIG/prednisone group in standard-risk pregnancies. In this study, fetal blood sampling, to evaluate effectiveness of therapy, was performed at 32 weeks' gestational age.

The adverse effects of IVIG use in maternal alloimmune thrombocytopenia are uncommon. Moderate to severe fatigue has been reported, and in only 1 out of 200 cases IVIG therapy was discontinued due to a rash. ${ }^{41}$ More adverse effects have been reported with prednisone and include gestational diabetes, fluid retention, mood swings, insomnia, and jitteriness. ${ }^{41}$ Bussel et $\mathrm{al}^{39}$ reported two cases of oligohydramnios in women who had received dexamethasone. In one case, the mother received three times the dose of dexamethasone; however, the oligohydramnios resolved following discontinuation of the steroid.

\section{Mode of Delivery}

There is no clear evidence on the mode of delivery for fetomaternal alloimmune thrombocytopenia, and cesarean section is performed for either maternal obstetric indications or the planned delivery of known high-risk cases. Van den Akker et $\mathrm{a}^{42}$ reported on 32 pregnancies affected by fetomaternal alloimmune thrombocytopenia; all mothers had delivered a previous sibling with NAIT but no ICH. Twenty-three infants were delivered vaginally, 3 of whom had severe thrombocytopenia. No infant had an ICH.

In summary, the antenatal management of pregnancies complicated by alloimmunization can be guided by an algorithmic, risk-based approach. ${ }^{34,35,40,41}$ Fetuses at highest risk are those who have a sibling who suffered an in utero ICH. There is an increasing trend to utilize noninvasive protocols in the management of alloimmune thrombocytopenia because of significant procedural complications following confirmatory and follow-up fetal blood sampling. Prospective, comparative studies indicate that the noninvasive approach may be both safe and effective, but the evidence remains inconclusive because of small sample sizes. ${ }^{34,35,40,41}$ In practice, IVIG $(1 \mathrm{~g} / \mathrm{kg})$ administered weekly from 24 weeks' gestation onward is the most common noninvasive protocol utilized. The management of all alloimmunized pregnancies should occur in a tertiary care center with immediate access to neonatal services. Treatment modalities available include in utero platelet antigen-compatible transfusions, maternal IVIG, and systemic steroids either singly or as combined therapy depending on the presence or absence of an $\mathrm{ICH}$ in a previous sibling. ${ }^{35,40-42}$ In the absence of placebo-controlled trials, the use of IVIG or steroids independently, in the antenatal period, stems from RCTs evaluating these therapies alone versus an alternative combined therapeutic intervention, which demonstrated safety of these maneuvers. Cordocentesis should be reserved for the experienced practitioner and to evaluate effectiveness of therapy.

\section{Postnatal Management}

There is no universal consensus on the treatment threshold for neonates presenting with NAIT at birth. A survey of Canadian and German neonatologists addresses this issue: $60 \%$ of Canadian neonatologists would commence treatment of preterm infants with a platelet count of 30 to $50 \times 10^{9} / \mathrm{L}$, whereas only $32 \%$ of the German neonatologists would start treatment at this level and $25 \%$ would use a threshold of 10 to $20 \times 10^{9}$ / L. In term infants, $6 \%$ of Canadian neonatologists and $16 \%$ of German neonatologists use a transfusion threshold level between 5 and $10 \times 10^{9} / \mathrm{L}^{43}$

Different transfusion thresholds appear throughout the literature, but they are not based on high-quality trials. A transfusion threshold of $<20 \times 10^{9} / \mathrm{L}$ or clinical bleeding has been proposed by Ouwehand et $\mathrm{al}^{44}$ and a threshold of $<30 \times 10^{9} / \mathrm{L}$ has been suggested by Blanchette et $\mathrm{al}^{10}$ and Arnold et al. ${ }^{29}$ Overall, a platelet count of $<30 \times 10^{9} / \mathrm{L}$ is generally accepted as a clinical trigger for therapeutic intervention. Bussel et $\mathrm{al}^{45}$ also suggested that the platelet count be kept above $100 \times 10^{9} / \mathrm{L}$ initially, if there was evidence of clinical bleeding, such as an intraventricular hemorrhage, and the platelet count should then be maintained at $>50 \times 10^{9} / \mathrm{L}$ for 1 to 2 weeks.

Several therapies are available for the clinician to treat the infant born with NAIT. However, as many of these infants present unexpectedly, at the time of delivery or shortly thereafter, the most recommended treatment option may not be readily available. An algorithm for the management of the unanticipated infant with NAIT is shown in Fig. 1.

Transfusion with matched antigen-negative platelet concentrates is the treatment of choice for an infant presenting with NAIT. HPA-1a- and HPA-5b-negative platelets are compatible in $95 \%$ of cases presenting with NAIT and produce higher increments in platelet counts with longer survival. ${ }^{44,46}$ However, few hospitals and national blood transfusion services have a registry of typed donors and stock such platelets for immediate transfusion. ${ }^{43}$ Compounding this issue is that physicians are often unaware that this treatment modality is available in their institutions. ${ }^{43}$

Apheresis, leukoreduced, plasma-depleted, irradiated, or washed maternal platelets is an alternative therapy. This strategy is of value for cases diagnosed in 
utero, as there is time to prepare the platelets prior to delivery. Platelet preparation takes time, as viral testing is mandatory prior to transfusion. Other treatment options are preferred for the unexpected severely thrombocytopenic infant.

Transfusion of random donor platelets has been shown to offer some benefit to infants with NAIT. ${ }^{47,48}$ However, although highly effective in some cases, this treatment modality should be used only to temporize the situation until compatible platelets are available. ${ }^{49,50}$

High-dose IVIG ( $400 \mathrm{mg} / \mathrm{kg}$ day over 5 days) has been shown to be effective in infants with NAIT in a small number of case reports. ${ }^{51-53}$ Ballin et $\mathrm{al}^{54}$ reported success with a dose of $1 \mathrm{~g} / \mathrm{kg} / \mathrm{d}$ for 2 consecutive days, and Linder et $\mathrm{al}^{55}$ reported a beneficial effect of a single dose of IVIG $(1 \mathrm{~g} / \mathrm{kg} / \mathrm{d})$ in four infants with a fifth infant requiring a second dose. These authors reported no adverse effects. Although some centers advocate the use of IVIG combined with either random donor or matched platelets, the efficacy of this strategy has not been evaluated prospectively.

\section{CONCLUSION}

The potentially devastating sequelae of alloimmune thrombocytopenia necessitate a rapid response from the health care team in regard to diagnosis and treatment. In the absence of large-scale, randomized controlled trials, there continues to be disparity among health care providers regarding the optimum management of FNAIT and NAIT. The risks and benefits of both maternal and fetal interventions need to be carefully weighed based on experience within institutions and evidence from current scientific literature. The diagnosis and care of the infant requires a coordinated, collaborative effort between obstetrics, neonatology, hematology, and transfusion medicine. Local, consensus protocols should be established to guide consistency in the treatment of NAIT. The infant born outside of a tertiary-level hospital with NAIT should be immediately transfused with random donor platelets, if severe thrombocytopenia exists or bleeding is evident, and promptly transferred to a tertiary level neonatal intensive care unit for further management.

\section{REFERENCES}

1. Burrows RF, Kelton JG. Fetal thrombocytopenia and its relation to maternal thrombocytopenia. N Engl J Med 1993; 329:1463-1466

2. Metcalfe P, Watkins NA, Ouwehand WH, et al. Nomenclature of human platelet antigens. Vox Sang 2003;85:240245

3. Gruel Y, Boizard B, Daffos F, Forestier F, Caen J, Wautier JL. Determination of platelet antigens and glycoproteins in the human fetus. Blood 1986;68:488-492
4. Blanchette VS, Chen L, de Friedberg ZS, Hogan VA, Trudel E, Décary F. Alloimmunization to the P1A1 platelet antigen: results of a prospective study. Br J Haematol 1990; 74:209-215

5. Doughty HA, Murphy MF, Metcalfe P, Waters AH. Antenatal screening for fetal alloimmune thrombocytopenia: the results of a pilot study. Br J Haematol 1995;90: 321-325

6. Durand-Zaleski I, Schlegel N, Blum-Boisgard C, Uzan S, Dreyfus M, Kaplan C; Immune Thrombocytopenia Working Group. Screening primiparous women and newborns for fetal/neonatal alloimmune thrombocytopenia: a prospective comparison of effectiveness and costs. Am J Perinatol 1996; 13:423-431

7. Kjeldsen-Kragh J, Killie MK, Tomter G, et al. A screening and intervention program aimed to reduce mortality and serious morbidity associated with severe neonatal alloimmune thrombocytopenia. Blood 2007;110:833-839

8. Turner ML, Bessos H, Fagge $T$, et al. Prospective epidemiologic study of the outcome and cost-effectiveness of antenatal screening to detect neonatal alloimmune thrombocytopenia due to anti-HPA-1a. Transfusion 2005; 45:1945-1956

9. Williamson LM, Hackett G, Rennie J, et al. The natural history of fetomaternal alloimmunization to the plateletspecific antigen HPA-1a (P1A1, Zwa) as determined by antenatal screening. Blood 1998;92:2280-2287

10. Blanchette VS, Johnson J, Rand M. The management of alloimmune neonatal thrombocytopenia. Baillieres Best Pract Res Clin Haematol 2000;13:365-390

11. Rayment R, Brunskill SJ, Stanworth S, Soothill PW, Roberts DJ, Murphy MF. Antenatal interventions for fetomaternal alloimmune thrombocytopenia. Cochrane Database Syst Rev 2005;(1 Issue 1):CD004226

12. Mueller-Eckhardt C, Mueller-Eckhardt G, Willen-Ohff H, et al. Immunogenicity of and immune response to the human platelet antigen Zwa is strongly associated with HLA-B8 and DR3. Tissue Antigens 1985;26:71-76

13. Davoren A, McParland P, Crowley J, Barnes A, Kelly G, Murphy WG. Antenatal screening for human platelet antigen-1a: results of a prospective study at a large maternity hospital in Ireland. BJOG 2003;110:492-496

14. Maslanka K, Guz K, Zupanska B. Antenatal screening of unselected pregnant women for HPA-1a antigen, antibody and alloimmune thrombocytopenia. Vox Sang 2003;85: 326-327

15. Dreyfus M, Kaplan C, Verdy E, Schlegel N, Durand-Zaleski I, Tchernia G; Immune Thrombocytopenia Working Group. Frequency of immune thrombocytopenia in newborns: a prospective study. Blood 1997;89:4402-4406

16. de Moerloose P, Boehlen F, Extermann P, Hohfeld P. Neonatal thrombocytopenia: incidence and characterization of maternal antiplatelet antibodies by MAIPA assay. Br J Haematol 1998;100:735-740

17. Uhrynowska M, Niznikowska-Marks M, Zupańska B. Neonatal and maternal thrombocytopenia: incidence and immune background. Eur J Haematol 2000;64:42-46

18. Ghevaert C, Campbell K, Walton J, et al. Management and outcome of 200 cases of fetomaternal alloimmune thrombocytopenia. Transfusion 2007;47:901-910

19. Bussel JB, Zabusky MR, Berkowitz RL, McFarland JG. Fetal alloimmune thrombocytopenia. N Engl J Med 1997; $337: 22-26$ 
20. Spencer JA, Burrows RF. Feto-maternal alloimmune thrombocytopenia: a literature review and statistical analysis. Aust N Z J Obstet Gynaecol 2001;41:45-55

21. Killie MK, Husebekk A, Kjeldsen-Kragh J, Skogen B. A prospective study of maternal anti-HPA 1a antibody level as a potential predictor of alloimmune thrombocytopenia in the newborn. Haematologica 2008;93:870-877

22. Skogen B, Husebekk A, Killie MK, Kjeldsen-Kragh J. Neonatal alloimmune thrombocytopenia is not what it was: a lesson learned from a large prospective screening and intervention program. Scand J Immunol 2009;70: 531-534

23. Radder CM, Brand A, Kanhai HHH. Will it ever be possible to balance the risk of intracranial haemorrhage in fetal or neonatal alloimmune thrombocytopenia against the risk of treatment strategies to prevent it? Vox Sang 2003;84: 318-325

24. Kanhai HHH, Porcelijn L, Engelfriet CP, et al. Management of alloimmune thrombocytopenia. Vox Sang 2007;93: 370-385

25. Kjeldsen-Kragh J, Husebekk A, Killie MK, Skogen B. Is it time to include screening for neonatal alloimmune thrombocytopenia in the general antenatal health care programme?. Transfus Apher Sci 2008;38:183-188

26. Fretheim A. Cost-effectiveness analysis of screening for neonatal alloimmune thrombocytopenia was based on invalid assumption. BJOG 2008;115:412-413; author reply 413414, discussion 414

27. Killie MK, Kjeldsen-Kragh J, Husebekk A, Skogen B, Olsen JA, Kristiansen IS. Cost-effectiveness of antenatal screening for neonatal alloimmune thrombocytopenia. BJOG 2007; 114:588-595

28. Kiefel V, Santoso S, Weisheit M, Müeller-Eckhardt C. Monoclonal antibody-specific immobilization of platelet antigens (MAIPA): a new tool for the identification of platelet-reactive antibodies. Blood 1987;70:1722-1726

29. Arnold DM, Smith JW, Kelton JG. Diagnosis and management of neonatal alloimmune thrombocytopenia. Transfus Med Rev 2008;22:255-267

30. Kaplan C, Daffos F, Forestier F, et al. Management of alloimmune thrombocytopenia: antenatal diagnosis and in utero transfusion of maternal platelets. Blood 1988;72:340-343

31. Overton TG, Duncan KR, Jolly M, Letsky E, Fisk NM. Serial aggressive platelet transfusion for fetal alloimmune thrombocytopenia: platelet dynamics and perinatal outcome. Am J Obstet Gynecol 2002;186:826-831

32. Daffos F, Forestier F, Muller JY, et al. Prenatal treatment of alloimmune thrombocytopenia. Lancet 1984;2:632

33. Nicolini U, Rodeck $\mathrm{CH}$, Kochenour NK, et al. In-utero platelet transfusion for alloimmune thrombocytopenia. Lancet 1988;2:506

34. Birchall JE, Murphy MF, Kaplan C, Kroll H; European Fetomaternal Alloimmune Thrombocytopenia Study Group. European collaborative study of the antenatal management of feto-maternal alloimmune thrombocytopenia. $\mathrm{Br} \mathrm{J} \mathrm{Haematol}$ 2003;122:275-288

35. van den Akker ES, Oepkes D, Lopriore E, Brand A, Kanhai $\mathrm{HH}$. Noninvasive antenatal management of fetal and neonatal alloimmune thrombocytopenia: safe and effective. BJOG 2007; 114:469-473

36. Paidas MJ, Berkowitz RL, Lynch L, et al. Alloimmune thrombocytopenia: fetal and neonatal losses related to cordocentesis. Am J Obstet Gynecol 1995;172(2 Pt 1): 475-479
37. Simon HU, Späth PJ. IVIG-mechanisms of action. Allergy 2003;58:543-552

38. Koleba T, Ensom MHH. Pharmacokinetics of intravenous immunoglobulin: a systematic review. Pharmacotherapy 2006; 26:813-827

39. Bussel JB, Berkowitz RL, Lynch L, et al. Antenatal management of alloimmune thrombocytopenia with intravenous $\gamma$-globulin: a randomized trial of the addition of lowdose steroid to intravenous $\gamma$-globulin. Am J Obstet Gynecol 1996;174:1414-1423

40. Berkowitz RL, Kolb EA, McFarland JG, et al. Parallel randomized trials of risk-based therapy for fetal alloimmune thrombocytopenia. Obstet Gynecol 2006;107:91-96

41. Berkowitz RL, Lesser ML, McFarland JG, et al. Antepartum treatment without early cordocentesis for standard-risk alloimmune thrombocytopenia: a randomized controlled trial. Obstet Gynecol 2007;110(2 Pt 1):249-255

42. van den Akker E, Oepkes D, Brand A, Kanhai HH. Vaginal delivery for fetuses at risk of alloimmune thrombocytopenia? BJOG 2006;113:781-783

43. Bassler D, Greinacher A, Okascharoen C, et al. A systematic review and survey of the management of unexpected neonatal alloimmune thrombocytopenia. Transfusion 2008; 48:92-98

44. Ouwehand WH, Smith G, Ranasinghe E. Management of severe alloimmune thrombocytopenia in the newborn. Arch Dis Child Fetal Neonatal Ed 2000;82:F173-F175

45. Bussel JB, Primiani A. Fetal and neonatal alloimmune thrombocytopenia: progress and ongoing debates. Blood Rev 2008;22:33-52

46. Mueller-Eckhardt C, Kiefel V, Grubert A, et al. 348 cases of suspected neonatal alloimmune thrombocytopenia. Lancet 1989;1:363-366

47. Kiefel V, Bassler D, Kroll H, et al. Antigen-positive platelet transfusion in neonatal alloimmune thrombocytopenia (NAIT). Blood 2006;107:3761-3763

48. Win N. Provision of random-donor platelets (HPA-1a positive) in neonatal alloimmune thrombocytopenia due to anti HPA-1a alloantibodies. Vox Sang 1996;71:130-131

49. Allen D, Verjee S, Rees S, Murphy MF, Roberts DJ. Platelet transfusion in neonatal alloimmune thrombocytopenia. Blood 2007;109:388-389

50. Bakchoul T, Sachs UJ, Wittekindt B, Sclösser R, Bein G, Santoso S. Treatment of fetomaternal neonatal alloimmune thrombocytopenia with random platelets. Pediatr Blood Cancer 2008;50:1293-1294

51. Derycke M, Dreyfus M, Ropert JC, Tchernia G. Intravenous immunoglobulin for neonatal isoimmune thrombocytopenia. Arch Dis Child 1985;60:667-669

52. Sidiropoulos D, Straume B. The treatment of neonatal isoimmune thrombocytopenia with intravenous immunoglobin (IgG i.v.). Blut 1984;48:383-386

53. Suarez CR, Anderson C. High-dose intravenous gammaglobulin (IVG) in neonatal immune thrombocytopenia. Am J Hematol 1987;26:247-253

54. Ballin A, Andrew M, Ling E, Perlman M, Blanchette V. High-dose intravenous gammaglobulin therapy for neonatal autoimmune thrombocytopenia. J Pediatr 1988;112: 789-792

55. Linder N, Shapiro SC, Moser AM, Roitman J, Engelhard D. Treatment of neonatal immune thrombocytopenia with high dose intravenous $\gamma$-globulin. Dev Pharmacol Ther 1990;14: 205-208 